\title{
PRESENCIA DEL GÉNERO BARRONOPSIS (ARANEAE: AGELENIDAE) EN LA HISPANIOLA CON LA DESCRIPCIÓN DE UNA ESPECIE NUEVA
}

\author{
Giraldo Alayón \\ Museo Nacional de Historia Natural de Cuba (MNHNCu). Obispo \# 61, Plaza de Armas, \\ La Habana, Cuba.moffly@informed.sld.cu
}

\section{RESUMEN}

Se describe una nueva especie de Barronopsis Chamberlin et Ivie, 1941, para La Hispaniola. Esta especie fue encontrada en la República Dominicana. Este es el primer registro de la familia Agelenidae y del género Barronopsis para La Hispaniola. Se incluye una breve discusión sobre las relaciones de esta especie con las ya descritas del área antillana.

Palabras clave: Araneae, Agelenidae, Barronopsis, taxonomía, especie nueva, La Hispaniola.

Title: Presence of the genus Barronopsis (Araneae: Agelenidae) in Hispaniola Island with description of a new species.

\section{ABSTRACT}

A new species of Barronopsis Chamberlin et Ivie, 1941 is described for Hispaniola. This species was found in the Dominican part of the island. This is the first record of the spider family Agelenidae and the genus Barronopsis for Hispaniola. A brief discussion of the relationships of this species with those already reported for the Antillean area is included

Key words: Araneae, Agelenidae, Barronopsis, taxonomy, new species, Hispaniola.

\section{INTRODUCCIÓN}

El género Barronopsis fue reportado, por primera vez, para Las Antillas Mayores por Alayón (1988) basado en especímenes colectados en la Reserva de la Biosfera "Cuchillas del Toa" (provincias Holguín-Guantánamo, Cuba). Posteriormente, en Alayón (1993), se describieron tres especies de este género para Cuba, dos de las cuales fueron sinonimizadas por Stocks (2009) en la última revisión del género. De acuerdo con Penney y Pérez-Gelabert (2002) y Penney (2008), hasta el presente no hay registro alguno de la familia Agelenidae para La Hispaniola, tanto en la fauna extinta (en ámbar) como en la actual. En este trabajo se reporta, por primera vez, a la familia Agelenidae y al género Barronopsis para La Hispaniola con la descripción de una especie nueva de los Parques Jaragua y Bahoruco y el registro de Barronopsis barrowsi (Gertsch) del Parque Nacional Valle Nuevo.

\section{OBJETIVO}

- Describir una nueva especie del género Barronopsis Chamberlin et Ivie, 1941 y discutir su relación con otras especies de Las Antillas.

\section{MATERIALES Y MÉTODOS}

Las mediciones (en $\mathrm{mm}$ ) se realizaron con micrómetro ocular de escala líneal, de acuerdo con los procedimientos explicados en Alayón García (1976). La disposición de las espinas fue 
descrita según Petrunkevitch (1925) y Platnick y Shadab (1975). Los genitales de las hembras fueron estudiados según la metodología de Levi (1965). Se utilizó, en la nomenclatura para la descripción de los genitales de machos y hembras, a Gering (1953) y Stocks (2009). En el ordenamiento clasificatorio del grupo se sigue a Roth y Brame (1972) y a Stocks (2009). Abreviaturas utilizadas: OMA, ojos medios anteriores; OLA, ojos laterales anteriores; OMP, ojos medios posteriores; OLP, ojos laterales posteriores; MNHNCu, Museo Nacional de Historia Natural de Cuba; MNHNSD, Museo Nacional de Historia Natural de Santo Domingo; CGA, Colección de Giraldo Alayón.

\author{
SISTEMÁTICA \\ Familia Agelenidae, C. L. Koch, 1837 \\ Subfamilia Ageleninae Simon, 1897 \\ Grupo Ageleneae Simon, 1897
}

Género Barronopsis Chamberlin et Ivie, 1941

Borde posterior del epigino sin cavidad copulatoria, con dos proyecciones medias en el margen anterior. Émbolo con tres o cuatro vueltas apretadas en la base, y una o más sueltas en el ápice. Cimbio con una concavidad opuesta al émbolo. Este taxon fue descrito como un subgénero de Agelenopsis por Giebel (1869), y elevado a la categoría de género por Lehtinen (1967).

\title{
Grupo de especies B. barrowsi
}

Machos con menos de dos vueltas en el émbolo, conductos espermáticos ondulados; hembras con las guías del atrio con los lóbulos anchos, angulados o ligeramente redondeados. Con una banda o línea blanca longitudinal en el esternón. Prosoma con menos de tres milímetros de longitud total. Telas en contacto con el suelo, frecuentes en agujas de pino, en hojas de palmas y montones de gramíneas. Especies que lo forman: B. barrowsi (Gertsch, 1934) y B. stephaniae Stocks, 2009.

Barronopsis pelempito sp. nov.

Figuras 1-3, 4, 5

Diagnosis. Los machos presentan las ramas pequeñas del conductor más finas, el extremo del émbolo más aguzado y el proceso tegular más grueso que en $B$. barrowsi. Las hembras con las prolongaciones del epigino más visibles, y las espermatecas más unidas a los tubos de fertilización que en B.barrowsi.

Diagnosis. Males with the branches of the conductor thinner and the tegular process more thickness than $B$. barrowsi. Females with the extensions of the epyginum more visible and the spermateci more joined to the fertilizations tubes than in B. barrowsi.

Holotipo. Macho holotipo de Hoyo de Pelempito, Parque Nacional Sierra de Bahoruco, Pedernales, República Dominicana, 18 05' lat. N, 71 30’ long. W, noviembre 15, 2003 (G. Alayón) en el MNHNCu.

Etimología. El nombre de la especie, en aposición, referido a la localidad tipo.

Descripción. Macho holotipo. Prosoma amarillo pálido, con dos manchas pardas laterales desde el área ocular hasta la parte torácica; área ocular pardo-oscura. Ligeramente convexo, 


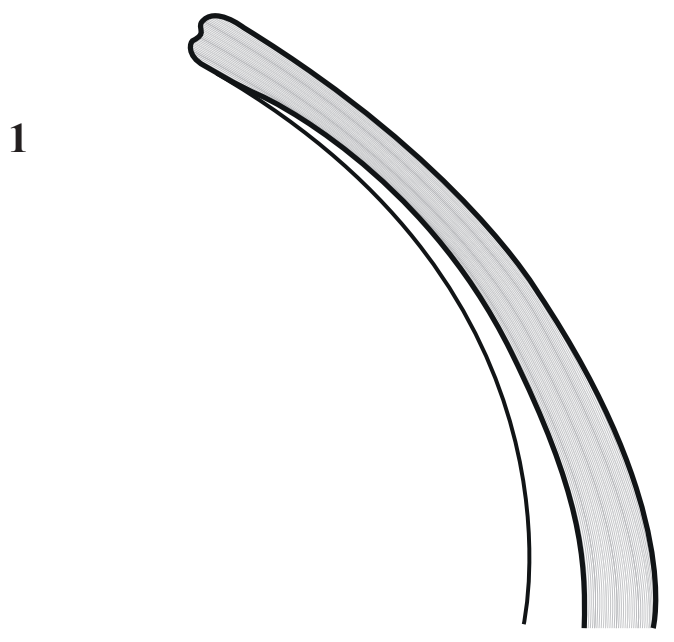

2
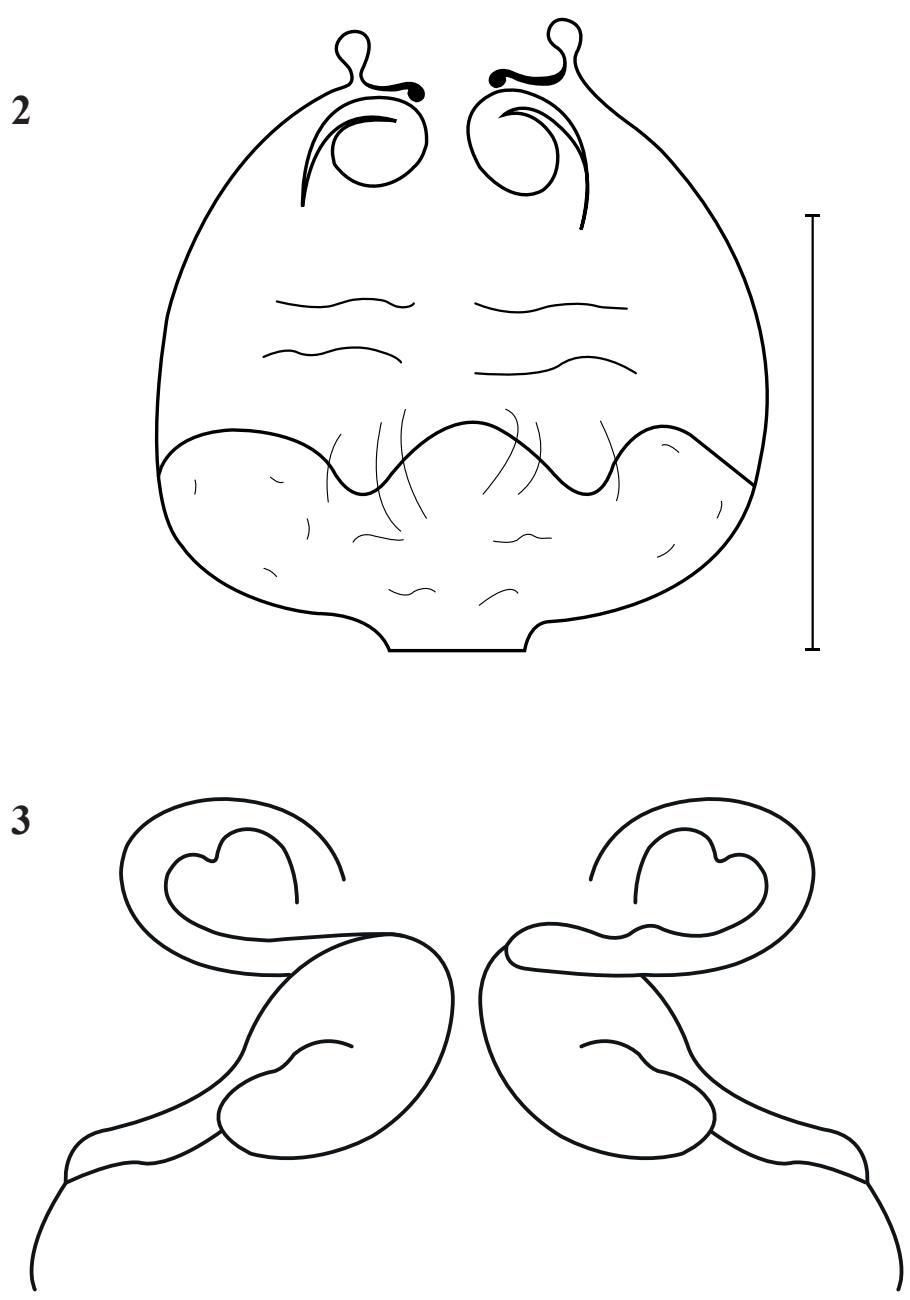

Figuras 1-3. Barronopsis pelempito sp. nov. 1, ápice del émbolo. 2, vista ventral del epigino (escala 0,5 mm). 3, vista dorsal del epigino. 
algo más elevado delante de la fóvea torácica (recta y poco visible); parcialmente cubierto de vellosidad recostada y oscura, con una línea de cerdas desde el clípeo hasta la parte posterior del área ocular; área ocular con vellosidad corta y recostada. Coxas amarillo-pálidas; labios y endites grisáceos con visos amarillentos. Esternón con cerdas espiniformes, más profusas en los márgenes; endites rectos con cerdas espiniformes. Tallos de los quelíceros pardo-oscuros con visos amarillentos. Promargen del surco ungueal con un dentículo, retromargen con tres dentículos; quelíceros con escasos pelos filiformes y fuertes cerdas en la parte interna y debajo de la garra del quelícero. Palpo, apófisis media membranosa con dos cúspides, una mayor y otra más pequeña, en la parte distal; proceso tegular arqueado hacia arriba, con el extremo en forma de cincel; conductor ancho y con dos ramas, la mayor en la parte superior; proceso externo de la tibia ancho y terminado en punta roma, con una elevación en la parte posterior; pars pendula visible en el extremos. Con cerdas espiniformes y pelos filiformes recostados, más profusos en los artejos de las patas anteriores con escasas tricobotrias dorsales, más abundantes en los artejos anteriores. Espinación de las patas: tibia I, V 1p-1p-1r-1p-0; D 0-0, P 1-1, R 0-0; metatarso I, V 2-2-2, D 0-0, P 1-0-0, R 1-0-0; tibia II, V 0-1r-1r-1p-0, D 0-0, P 0-0, R 0-0; metatarso II, V 2-2-2, D 1-1, P 0-0-0, R 1-0-0; tibia III, V 1p-1p-0-0-0, D 1-0, P 1-1, R 1-0; metatarso III, V 2-2-2, D 1-1, P 1-0-0, R 1-1-0; tibia IV, V 1p-1p-0-0-2, D 1-1, P 1-0, D 1-1; metatarso IV, V 2-2-2, D 1-1, P 1-1-1, R 1-1-1. Opistosoma con las partes laterales posteriores más oscuras que las dorsales y anteriores; parte dorsal con dos bandas claras quebradas y paralelas en la parte anterior pero que se unen en la parte posterior; parte ventral amarillo pálido, con dos líneas pardo-oscuras paralelas desde debajo de la zona epigástrica hasta 2/3 del mismo, con cuatro marcas separadas que se unen en la parte anterior de las hileras (de color amarillo- grisáceas). Medidas (en mm.): longitud del prosoma, 2.75; anchura del prosoma, 1.85; longitud del esternón, 1.35; anchura del esternón, 1.20; longitud del opistosoma, 3.25; longitud total, 6.00; OMA, 0.175; OLA, 0.150; OMP, 0.125; OLP, 0.150; fémur I, 32.0; tibia I, 2.35; fémur II, 2.65; tibia II, 2.35; fémur III, 2.60; tibia III, 2.10; fémur IV, 3.40; tibia IV, 2.90. Fórmula de las patas: 4123.

Descripción. Hembra paratipo. Los colores son más acentuados que en los machos, aunque el patrón de colorido es similar con la excepción de que en la parte dorsal del opistosoma las dos bandas claras y quebradas permanecen paralelas. La pilosidad en el cuerpo y patas es similar. Espinación de las patas: tibia I, V 1p; metatarso I, V 0-1r-2-2; D 0; tibia II, V0; metatarso II, V 0-1r-1-1; D0; tibia III, V 1p; metatarso III, V 1p-0-2-2; D0; tiba IV, V 0; metatarso IV, V 1p-02-2; D 1. Epigino con los rebordes suaves; atrio amplio y alargado, de forma piramidal; a través del tegumento se ven las espermatecas; vulva con los divertículos unidos a la parte posterior de la bursa copulatrix (membanosas en la parte anterior), por un fino conducto esclerosado, pegados a la superficie interior de las espermatecas, éstas, de forma arriñonada, se encuentran casi unidas, en posición posterior, con las paredes externas muy exclerosadas. Medidas (en mm.): longitud del prosoma, 2.90; anchura del prosoma, 2.00; longitud del esternón, 1.30; anchura del esternón, 1.05; longitud del opistosoma, 4.05; longitud total, 6.95; OMA, 0.175; OLA, 0.175; OMP, 0.125; OLP, 0.125; fémur I, 3.00; tibia I, 2.70; fémur II, 2.45; tibia II, 2.30; fémur III, 2.0; tibia III, 1.75; fémur IV, 3.25; tibia IV, 3.15. Fórmula de las patas: 4123.

Variación. Las hembras varían entre 6.40- $7.35 \mathrm{~mm}$ de longitud total. Un macho (paratipo) medía $5.20 \mathrm{~mm}$ de longitud total. Los especímenes de la localidad "Carretera de Aceitillar- Pedernales" son de colores más pálidos. En las hembras la forma del reborde superior del epigino es variable (más aguzado en algunas); en la vulva es variable la posición de los divertículos.

Distribución. Desde la Sierra de Bahoruco hacia el Sur, hasta la Isla Beata (República Dominicana).

Historia Natural. De esta especie hemos encontrado tres poblaciones a diferentes alturas sobre el nivel del mar, las que se encuentran en la localidad tipo (Hoyo de Pelempito) construyen sus 
telas entre las agujas de pino que han caído al suelo y entre las hojas de los agaves; la que se encuentra en la localidad de "Carretera de Aceitillar-Pedernales" en arbustos bajos, utilizando de soporte parte de las hojas secas que caen; las de la Isla Beata construyen sus telas en los cactus.

Material examinado. Una hembra (paratipo), Isla Beata, Parque Nacional Jaragua, provincia Pedernales, República Dominicana, febrero 6, 1999 (Kennida Polanco, MNHNSD). Un macho (paratipo), Carretera Aceitillar-Pedernales, República Dominicana, Febrero 5, 2002, $18^{\circ} 34^{\prime}$ lat N, 71² $28^{\prime}$ long. W (Giraldo Alayón, CGA). Una hembra (paratipo), Hoyo de Pelempito, Parque Nacional Sierra de Bahoruco, provincia Pedernales, República Dominicana, febrero 3, 2002 (Giraldo Alayón, CGA). Dos hembras (paratipos), Carretera Aceitillar-Pedernales, República Dominicana, febrero 5, 2002 (Giraldo Alayón, MNHNCu). Dos hembras (paratipos), Hoyo de Pelempito, Parque Nacional Sierra de Bahoruco, provincia Pedernales, República Dominicana, noviembre 15, 2003 (Giraldo Alayón, MNHNSD). Dos hembras (paratipos), Carretera Aceitillarprovincia Pedernales, febrero 5, 2002 (Giraldo Alayón, MNHNCu). Tres hembras (paratipos), Hoyo de Pelempito, Parque Nacional Sierra de Bahoruco, provincia Pedernales, República Dominicana, noviembre 15, 2003 (Giraldo Alayón, MNHNCu). Una hembra (paratipo), Playa del Coco, Isla Beata, Parque Nacional Jaragua, provincia Pedernales, República Dominicana, marzo 19, 1999 (L. F. de Armas y Kennida Polanco, MNHNCu).

\section{Barronopsis barrowsi (Gertsch,1934)}

Figura 4

Distribución. En Stocks (2009) se considera a esta especie distribuida en varias localidades en el estado de Florida (EUA) y en la Reserva de la Biosfera "Cuchillas del Toa" en la provincia de Guantánamo (Cuba); en este trabajo se registra en el Parque Nacional Valle Nuevo, provincia La Vega, República Dominicana.

Historia Natural. En la nueva localidad se ha colectado en el interior de viviendas humanas, ha construido su tela tubular en oquedades de la pared; bajo cortezas semi-desprendidas en árboles y en el pajonal. También se ha colectado directamente en los pajones (Danthonia domingensis) y es común ver sus telas en la pequeña sabana donde hubo cultivo de papas frente a La Pirámide.

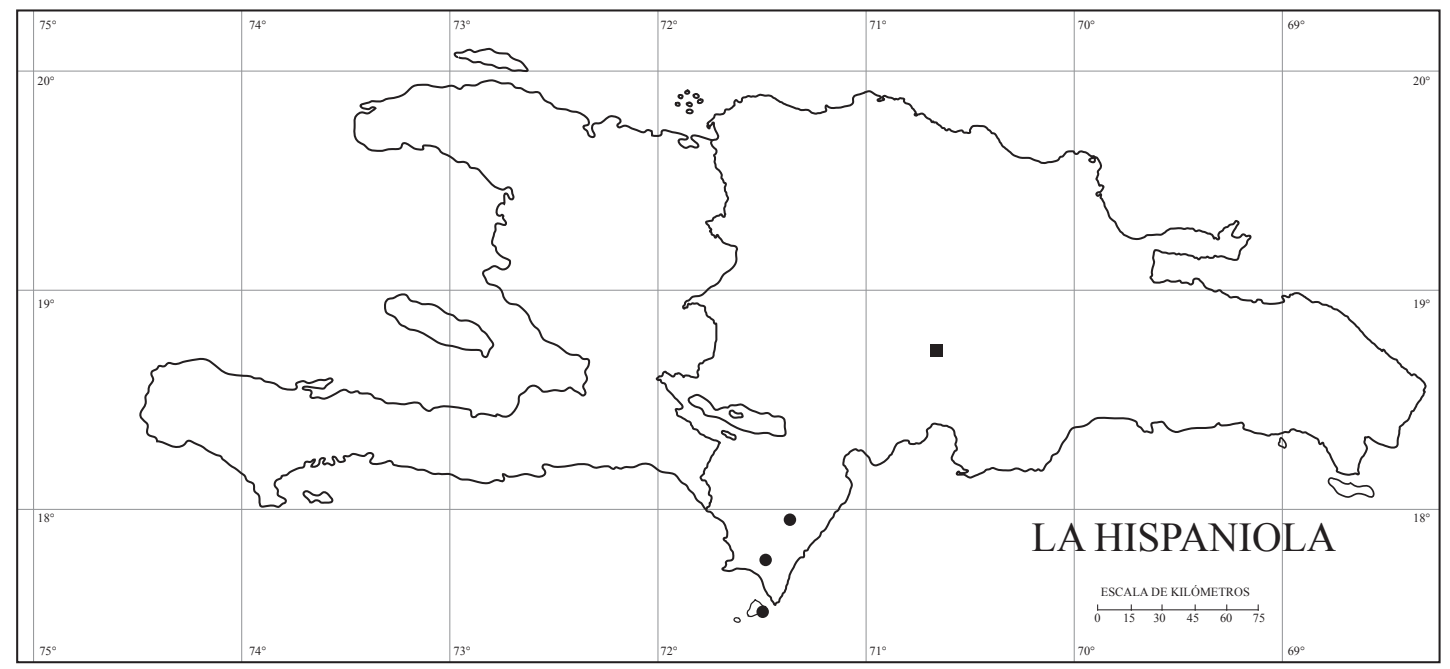




\section{DISCUSIÓN}

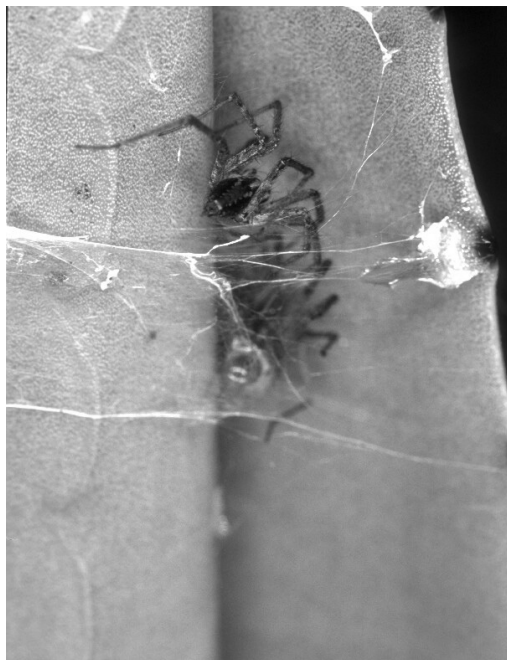

Figura 5. Hembra y macho de Barronopsis pelempito sp. nov., en hoja de Agave.
La araneofauna de La Hispaniola no está bien conocida (Penney, 1999, 2008; Penney y Pérez-Gelabert, 2002; Platnick y Penney, 2004), criterio que compartimos como resultado preliminar de nuestras colectas en varias regiones y parque nacionales de República Dominicana en los últimos años. La familia Agelenidae no está bien representada en Las Antillas Mayores, ya que de acuerdo con Platnick (2011) cuenta con tres especies, todas pertenecientes al género Barronopsis, y Tegenaria insularis Walckenaer, 1842 ha sido considerada insertae sedis (Roth 1967, 1968) y nomina dubia (Platnick, 2011). El hallazgo de dos especies pertenecientes a este género en La Hispaniola es un descubrimiento interesante, ya que tanto la familia como el género no han sido registrados en la fauna fósil (del ámbar) de dicha isla. Somos de la opinión que es muy probable que tanto el género como la familia estén mejor representados en esta isla antillana y que su fauna actual cuente con otros táxones aún por descubrir.

Según Roth (1954), el género Barronopsis es muy uniforme en sus caracteres morfológicos, lo cual puede corroborarse en la similitud de los genitales femeninos y masculinos en las diferentes especies, además, en Norteamérica algunas especies tienen una distribución simpátrica, lo cual no se cumple para las especies registradas para Cuba cuya distribución acusa una franca alopatría (Alayón García, 1993) y sólo se encuentran representadas en tres provincias [Pinar del Río, Artemisa, Guantánamo y un municipio especial, Isla de la Juventud (antigua Isla de Pinos) en el Archipiélago de los Canarreos, con la especie Barronopsis jeffersi, que es la de más amplia distribución en Cuba]. Tampoco es así para las registradas para República Dominicana, ya que $B$. barrowsi y $B$. pelempito sp. n. tienen una distribución alopátrica.

Barronopsis pelempito sp. nov. se encuentra muy relacionada con B. barrowsi, aunque la estructura del palpo y la forma y disposición de las espermatecas las separan. El grupo de especies $B$. barrowsi debe tener su origen en la zona Neártica, con propágulos del mismo invadiendo Las Antillas vía Cuba; este grupo se diversificó en este territorio y un segmento del mismo (probablemente el más oriental) invadió posteriormente a La Hispaniola, seguido de un proceso posterior de diversificación.

\section{AGRADECIMIENTOS}

Agradezco a Yvonne Arias, Sixto Incháustegui y Héctor Andújar, del Grupo Jaragua, las múltiples atenciones durante nuestras expediciones a República Dominicana; a Celeste Mir y Carlos Suriel la posibilidad de participar en el proyecto "Ecología de la fauna asociada al suelo en la sabana de pajón del Parque Nacional Valle Nuevo y su respuesta a los efectos del fuego y la agricultura" del Museo Nacional de Historia Natural de Santo Domingo, financiado por FONDOCYT, del Ministerio de Educación Superior, Ciencia y Tecnología (MESCyT) (232010); a Kennida Polanco parte del material estudiado y su compañía en varias salidas al campo; a Víctor González el apoyo en los estudios de la fauna caribeña; a Matilde Mota la gestión de los permisos para el acceso a las áreas naturales en el territorio dominicano; a Solanlly Carrero y Gabriel de los Santos, del Museo Nacional de Historia Natural de Santo Domingo, su gran ayuda en el trabajo de laboratorio y a Laura Watson por las fotos del macho y la hembra de Barronopsis pelempito sp. nov. 


\section{LITERATURA CITADA}

Alayón García, G.1976. Nueva especie de Nops MacLeay, 1893 (Araneae: Caponiidae) en Isla de Pinos, Cuba. Poeyana., 148: 1-6.

Alayón García, G. 1988. Lista preliminar de las arañas (Araneae) de la Reserva de la Biosfera Cuchillas del Toa, provincias Holguín- Guantánamo. Garciana., 11: 2-4.

Alayón García, G.1993. El género Barronopsis (Araneae: Agelenidae) en Cuba. Poeyana., 435: $1-16$.

Chamberlin, R.V. \& Ivie. 1941. North American Agelenidae of the genera Agelenopsis, Calilena, Ritalena, and Tortolena. Ann. Entomol. Soc. Amer., 34: 585-628.

Gering, R. L. 1953. Structure and function of the genitalia in some agelenid spiders. Smithsonian Misc. Coll., 121(4): 1-84.

Giebel, C. G. 1869. Veber einige Spinnen aus Illinois. Zeitschr. Ges. Naturwiss., 33: 248-253.

Lehtinen, P. T. 1967. Classification of the cribellate spiders and some allied families, with notes on the evolution of the suborder Araneomorphae. Ann. Zool. Fennici., 4: 199-468.

Levi, H. W. 1965. Techniques for the study of spider genitalia. Psyche, 72(2): 152-158.

Penney, D. 1999. Hypothesis for the Recent Hispaniolan spider fauna based on the Dominican Republic amber spider fauna. J. Arachnol., 27: 64-70.

Penney, D. 2008. Dominican Amber Spiders: A comparative paleontological-neontological approach to identification, faunistics, ecology and biogeography. Siri Scientific Press, London., 176 pp.

Penney D. y D. E. Pérez-Gelabert 2002. Comparison of Recent and Miocene Hispaniolan Spider faunas. Rev. Ibérica Aracnol., 6: 203-223.

Petrunkevitch, A.1925. Arachnida from Panama. Trans. Connecticut Acad. Arts Sci., 27: 51-248.

Platnick, N. I. 2011. The World Spider Catalog, (version 12.0). Online at, htpp://research.amnh. org./entomology/spider/catalog/INTRO2.html.

Platnick, N. I. y D. Penney 2004. A revision of a widespread genus Zimiris (Araneae: Prodidomidae). Amer. Mus. Novitat., 3450: 1-12.

Platnick, N. I. y M. U. Shadab. 1975. A revision of the spider genus Gnaphosa (Araneae: Gnaphosidae) in America. Bull. Amer. Mus. Nat. Hist., 155(1): 1-66.

Roth, V. D. 1954. The subgenus Barronopsis (Arachnida, Agelenidae, Agelenopsis). Amer. Mus. Novitat., 1678: 1-7.

Roth, V. D. 1967. A review of the South American spiders of the family Agelenidae (Arachnida: Araneae). Bull. Amer. Mus. Nat. Hist., 134(5): 299-345.

Roth, V. D. 1968. The spider genus Tegenaria in the Western Hemisphere (Agelenidae). Amer. Mus. Novitat. 2323: 1-33. 
Roth, V. D. y P. L. Brame 1972. Nearctic genera of the spider family Agelenidae (Arachnida, Araneida). Amer. Mus. Novitat., 2505: 1-52.

Stocks, I. C. 2009. Systematics and Natural History of Barronopsis (Araneae: Agelenidae), with descriptions of a new species. Zootaxa, 2270: 1-38.

[Recibido: 10 de agosto, 2011. Aceptado para publicación: 21 de diciembre, 2011] 